\title{
Study on the Determination of Tauroursodeoxycholic Acid in Bear Bile Powder by HPLC-MS/MS
}

\author{
Kaibin Huang", Wenbi Pan, Chuntian Yang, Yingying Ye, Xiaoce Lyu \\ Public Technical Service Center, Wenzhou Institute of Industry and Science, Wenzhou 325028, Zhejiang, China \\ Email: kaibin.huang@163.com
}

\begin{abstract}
Objective - To establish a high performance liquid chromatography-tandem mass spectrometry (HPLC-MS/ MS) method for the rapid determination of tauroursodeoxycholic acid (TUDCA) in bear bile powder. Method — The chromatographic conditions were optimized. A Hypersil GOLD chromatographic column (Thermo Fisher, $100 \mathrm{~mm} \times 2.1$ $\mathrm{mm})$ was used with the mobile phase of methanol:water $(0.1 \mathrm{mmol} / 1$ ammonium acetate $+0.1 \%$ formic acid $)=75: 25$ for isocratic elution; the column temperature is $30.0^{\circ} \mathrm{C}$; the flow rate is $0.2 \mathrm{~mL} / \mathrm{min}$; the injection volume is $5 \mathrm{uL}$; the negative ion scanning mode and the select reaction monitoring are used. Through the compound optimization function of the instrument, the two characteristic ion pairs of TUDCA are m/z: 498.5/124.0 and m/z: 498.5/79.8 (quantitative ion pair). Results - The detection limit of this method was $5 \mathrm{ng} / \mathrm{mL}$, and the linear range was $20 \sim 1000 \mathrm{ng} / \mathrm{mL}$ (r=0.9993). The same sample was measured in parallel with the high performance liquid chromatography (HPLC/UV) method, and the analysis results were basically the same. Conclusion - Compared with HPLC/UV, HPLC-MS/MS is simple, more sensitive and accurate for the determination of TUDCA in bear bile powder. The method can be used in the quality assessment and process control of bear bile powder.
\end{abstract}

Keywords: HPLC-MS/MS, tauroursodeoxycholic acid (TUDCA), bear bile powder

\section{Introduction}

Bear bile powder is a kind of irregularly fragmented powder particles, crisp, brownish yellow, greenish yellow or dark brown, with glass-like luster, it is blindly precious Chinese medicinal material. TUDCA is the main active ingredient of bear bile powder. Its chemical name is $3 \alpha, 7 \beta$ dihydroxycholanoyl-N-taurine, which is a combined bile acid formed by the shrinkage between the shuttle group of ursodeoxycholic acid (UDCA) and the amino group of taurine. The molecular weight is 499.70. Bear bile powder has the effects of clearing away heat, calming liver, dissolving gallstones, and has strong analgesic, antispasmodic and anti-inflammatory effects. According to the National Drug Standard WS $-09(\mathrm{~B}-09)-96(\mathrm{Z})$ of National Medical Products Administration, the content of TUDCA in bear bile powder shall not be less than 23\%. At present, TUDCA in bear bile powder is mainly determined by high performance liquid chromatography (HPLC) ${ }^{[1][2][3]}$. Another major component present in bear bile powder is taurochenodeoxycholate acid (TCDCA). In this paper, the conditions of liquid chromatography are optimized so that TUDCA and TCDCA were completely separated. The mass spectrometer uses negative ion scanning mode, through the compound optimization function of the instrument, the precursor of TUDCA is [M$\mathrm{H}]-498.5$, and the SRM is used to monitor the two precursor/products $\mathrm{m} / \mathrm{z} 498.5 / 124.0, \mathrm{~m} / \mathrm{z} 498.5 / 79.8$, and $\mathrm{m} / \mathrm{z} 498.5 / 79.8$ is selected as the quantitative ion pair. Before sample detection, the blank sample is negative without interference. If a chromatographic peak appears in the TIC chart of the sample to be tested, its retention time is compared with the retention time of the chromatographic peak of the TUDCA reference substance, and the relative error is within $\pm 2 \%$. Two pairs of characteristic ion pairs appear in the mass spectrum, and the relative error between the selected relative ion abundance ratio and the relative ion abundance ratio of the TUDCA reference substance is within $\pm 25 \%$, then the chromatographic peak is determined to be TUDCA. The standard curve method was used to quantitatively calculate the content of TUDCA in the sample. The accuracy of this method was verified by comparing with the results determined by HPLC/UV method. Compared with the HPLC method, the HPLC-MS/MS method adopts the SRM mode, which only detects one product ion, which can effectively reduce the interference of the matrix. The sample does not require a complicated pre-treatment process, so this method is simple and suitable for the determination of TUDCA in bear bile powder. Further, because this method has a lower detection limit, specificity and high sensitivity, it is especially suitable for samples with very low content of TUDCA in drugs with appropriate sample pretreatment. 


\section{Experimental part}

\subsection{Apparatus and reagents}

Ultimate3000 Liquid Chromatography/TSQ Quantis Triple Quadrupole Mass Spectrometer (Thermo Fisher Scientific, USA)

Waters 1525 Liquid Chromatograph / Waters 2489 UV/Visible Light Detector (Waters Company, USA)

Sodium tauroursodeoxycholate standard product, the content is 93.4\% (batch number: 110816-201509, China Institute for Food and Drug Control 20mg)

Sodium taurochenodeoxycholate standard product, for identification (batch number: 110848-201007, China Institute for Food and Drug Control 20mg)

Bear bile powder (batch number: 201907003, Zhanxintang, 0.1g/bottle)

Bear bile powder (batch number: 201912003, Dianxiong, 0.3g/bottle)

Bear bile raw juice freeze-dried powder (Guanhuo bear farm, $0.1 \mathrm{~g} / \mathrm{bottle}$ )

Formic acid (chromatographically pure, Aladdin)

Ammonium acetate (chromatographically pure, Aladdin)

High purity water: resistivity $\geq 18.4 \mathrm{M} \Omega . \mathrm{cm}$

\subsection{Preparation of standard solution}

Accurately weigh $10 \mathrm{mg}$ of the standard sodium tauroursodeoxycholate, dissolve it in methanol, prepare a $1 \mathrm{mg} / \mathrm{mL}$ sodium tauroursodeoxycholate stock solution, and store it in a refrigerator at $4^{\circ} \mathrm{C}$ for later use. Use methanol to further dilute it into a standard solution of appropriate concentration.

Accurately weigh $10 \mathrm{mg}$ sodium taurochenodeoxycholate standard product, dissolve it in methanol, prepare $1 \mathrm{mg} / \mathrm{mL}$ sodium taurochenodeoxycholate stock solution, and store it in a refrigerator at $4^{\circ} \mathrm{C}$ for later use. Use methanol to further dilute it into a standard solution of appropriate concentration.

\subsection{Sample preparation}

\subsubsection{HPLC-MS/MS method sample pretreatment}

Weigh 10mg of bear bile powder accurately, dissolve it with a proper amount of methanol by using a ultrasonic cleaning machine, make the volume $10 \mathrm{~mL}$, take $1.5 \mathrm{~mL}$, filter with a 0.22 um nylon filter into a sample bottle, and wait for testing.

Use methanol as a blank sample.

\subsubsection{HPLC/UV method sample pretreatment}

Weigh 10mg of bear bile powder accurately, dissolve it with a proper amount of methanol by using a ultrasonic cleaning machine, make a constant volume of $10 \mathrm{~mL}$, take $1.5 \mathrm{~mL}$, filter with a $0.22 \mathrm{um}$ nylon filter, put it in a sample bottle, and put it on the instrument for testing.

\subsection{Experimental conditions}

\subsubsection{HPLC-MS/MS method instrument conditions}

HPLC-MS/MS method liquid phase conditions: chromatographic column adopts Hypersil GOLD chromatographic column (Thermo Fisher, $100 \mathrm{~mm} \times 2.1 \mathrm{~mm}$ ); column temperature: $30.0^{\circ} \mathrm{C}$; mobile phase: methanol:water $(0.1 \mathrm{mmol} / \mathrm{ml}$ ammonium acetate +1$) \%$ formic acid) $=75: 25, \mathrm{WV}$ isocratic elution; Flow rate: $0.2 \mathrm{~mL} / \mathrm{min}$; Injection volume: $5 \mathrm{uL}$.

HPLC-MS/MS method mass spectrometry conditions: negative ion scanning mode, Heat Electrospray Ionization (HESI); quantitative analysis by SRM; spray voltage: $4872 \mathrm{~V}$; vaporizer temperature: $245^{\circ} \mathrm{C}$; ion transfer tube temperature: $300^{\circ} \mathrm{C}$; sheath gas (Arb): 35; aux gas (Arb): 6.9; sweep gas (Arb): 1. M/z498.5/124.0 and m/z498.5/79.8 are used as the qualitative ion pairs, and $\mathrm{m} / \mathrm{z} 498.5 / 79.8$ is the quantitative ion pair.

\subsubsection{Chromatographic conditions of HPLC/UV method}

HPLC/UV method liquid phase conditions ${ }^{[4]}$ : Waters Symmetry C18 column (5um, 4.6×250mm); methanol:0.02M potassium dihydrogen phosphate buffer solution $(\mathrm{pH}=4.0$ adjusted with phosphoric acid) $=75: 25$ as the mobile phase; detection wavelength is $205 \mathrm{~nm}$; column temperature: $30^{\circ} \mathrm{C}$; flow rate: $1.0 \mathrm{ml} / \mathrm{min}$; injection $10 \mathrm{uL}$ for determination; record the chromatogram and calculate the content. 


\section{Results and discussion}

\subsection{Optimization of instrument conditions}

In the HPLC-MS/MS method, since TUDCA and TCDCA have similar structures and high polarity, a Hypersil GOLD column with strong retention of polar compounds is selected. This series of chromatographic columns are based on high-purity silica gel, which is a new type of chromatographic column that can provide perfect peak shape, narrow chromatographic peaks and better column efficiency. During the experiment, a mixed standard solution of TUDCA and TCDCA at a concentration of $100 \mathrm{ng} / \mathrm{mL}$ was prepared, and methanol/water and acetonitrile/water were used as mobile phases. It was found that acetonitrile/water could not achieve TUDCA and TCDCA separated effectively. Using methanol: water $(0.1 \mathrm{mmol} / \mathrm{L}$ ammonium acetate $+0.1 \%$ formic acid $)=75: 25$ isocratic elution method, the best separation of TUDCA and TCDCA can be achieved. By comparing with the chromatograms of TUDCA and TCDCA single-standard solutions, the retention time (RT) of TUDCA is $2.53 \mathrm{~min}$, and the retention time (RT) of TCDCA is $4.17 \mathrm{~min}$, and the two peaks are separated well (Figure 1).

TUDCA has poor high temperature stability. In the process of optimizing the vaporizer temperature of the mass spectrometer, it was found that a lower vaporizer temperature $\left(245^{\circ} \mathrm{C}\right)$ can make the instrument have better sensitivity and precision.

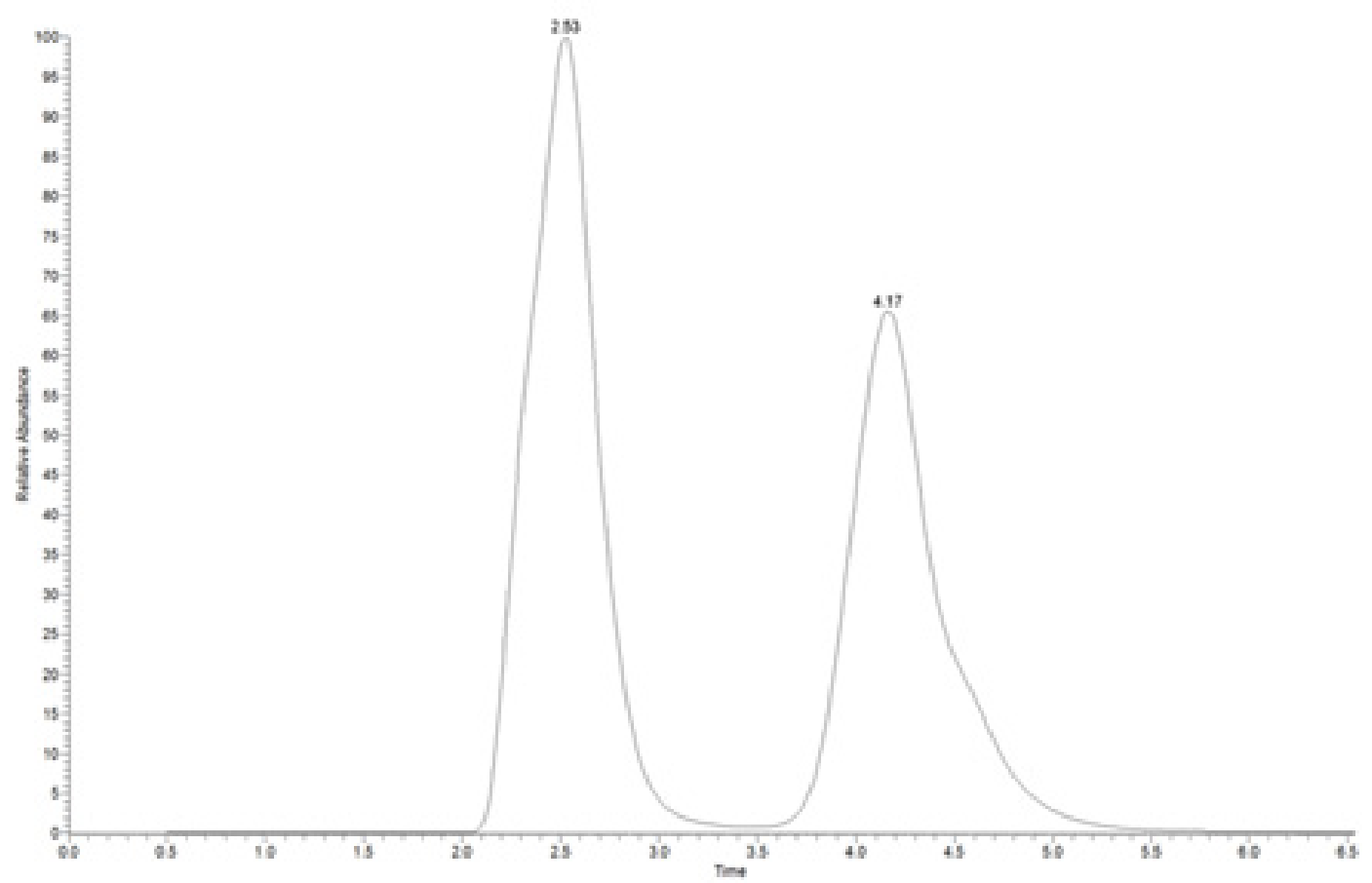

Figure 1. The TIC chart of TUDCA and TCDCA

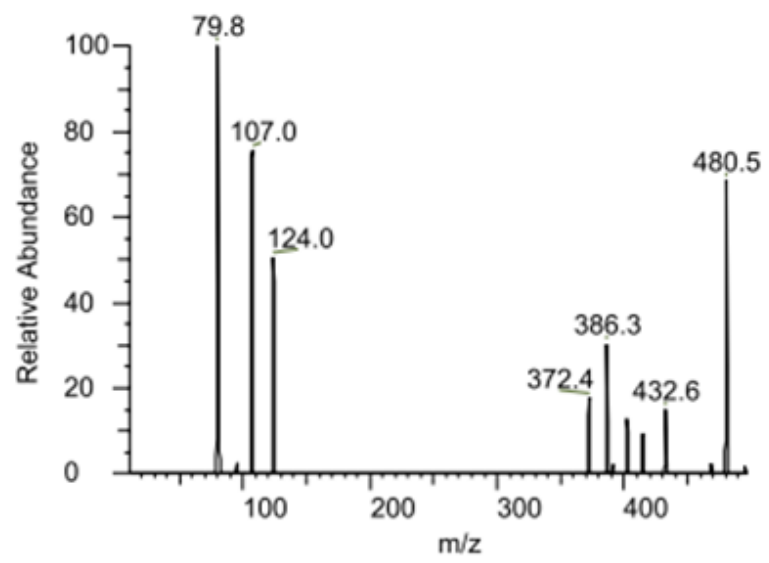

Figure 2. Mass spectrum of TUDCA 


\subsection{Detection limit, standard curve and linear range}

The TUDCA reference solution was gradually diluted to a suitable concentration, and the injection concentration (ng/ $\mathrm{mL}$ ) at a signal-to-noise ratio of 3:1 was used as the detection limit. The detection limit of the TUDCA was determined to be $5 \mathrm{ng} / \mathrm{mL}$.

Standard curve and concentration range: accurately measure the appropriate amount of TUDCA reference substance stock solution, and dilute it with methanol into a series of standard solutions with 7 concentration gradients of 20, 50, 100, $200,400,600$, and $1000 \mathrm{ng} / \mathrm{mL}$; use the concentration of TUDCA as the abscissa and the peak area as the ordinate to perform linear regression to obtain the regression equation. The linear equation of TUDCA is: $y=157.53 x+1992.3$; the linear range is $20-1000 \mathrm{ng} / \mathrm{mL}$; the correlation coefficient is 0.9993 in the concentration range; the linear relationship is good.

\subsection{Precision test}

Take the TUDCA stock solution prepared according to the method 2.2 and dilute it into a standard solution with a concentration of $100 \mathrm{ng} / \mathrm{mL}$. According to the above chromatographic conditions, inject 6 times continuously. Record the peak area of the chromatogram, and calculate the RSD of each peak area of TUDCA to be $2.16 \%$, indicating that the precision of the instrument is good.

\subsection{Repeatability test}

Take the same bear bile powder sample (batch number: 201907003), and prepare 6 test solution of bear bile powder in parallel according to the method 2.3.1. The samples were injected under HPLC-MS/MS conditions, and the average content of TUDCA measured was $29.9 \%$, and the RSD was $2.01 \%$, indicating that the method has good repeatability.

\subsection{Stability test}

Take a sample of bear bile powder (batch number: 201907003), and prepare a test solution of bear bile powder according to the method 2.3.1. The sample was injected every 2 hours within $0-24 \mathrm{~h}$, and the RSD calculated by the peak area was $2.21 \%$, indicating that the test solution of bear bile powder was stable within 24 hours.

\subsection{Recovery rate test}

Take the same bear bile powder sample (batch number: 201907003) according to method 2.3.1, and prepare 6 copies of bear bile powder test solution in parallel. Accurately measure $1 \mathrm{~mL}$ and add 200ng of TUDCA respectively, prepare 6 test solutions in parallel. The 6 samples were tested by HPLC-MS/MS method, determined by external standard method and the average recovery rate of TUDCA reference substance stock solution determined by external standard method was $101.2 \%$, and the RSD was $2.07 \%$.

\subsection{Comparison of the determination results of HPLC/UV method and HPLC-MS/MS method}

Three brands of freeze-dried bear bile powder samples were purchased from the market, and TUDCA content was determined by HPLC/UV and HPLC-MS/MS respectively. The results are shown in Table 1.

\begin{tabular}{ccc}
\multicolumn{3}{c}{ Table 1. TUDCA content in the samples } \\
\hline Sample & HPLC/UV (\%) & HPLC-MS/MS (\%) \\
\hline Bear bile powder batch number: 201907003 & 30.6 & 29.9 \\
Bear bile powder batch number: 201912003 & 35.9 & 35.4 \\
Bear bile juice freeze-dried powder & 31.4 & 31.2 \\
\hline
\end{tabular}

Compare the measurement results of the two methods, and there is no significant difference between the two.

\section{Conclusion}

In this paper, high performance liquid chromatography-tandem triple quadrupole mass spectrometry (HPLC-MS/MS) was developed to determine the content of tauroursodeoxycholic acid in bear bile powder, and the liquid phase conditions and mass spectrometry conditions were optimized. Simultaneously measure the same sample and compare and analyze the results. The results show that the HPLC-MS/MS method adopts the select reaction monitoring (SRM) for analysis. Two characteristic ion pairs are used for qualitative analysis, and one of the ion pairs is used for quantification, which can effectively reduce the influence of the matrix and has higher sensitivity, specificity, easy operation and good accuracy. 


\section{References}

[1] Li Jiaquan, Zheng Kunfang. Determination of tauroursodeoxycholic acid in bear bile by HPLC method. Yunnan Journal of Traditional Chinese Medicine and Materia Medica. 2016; 37(10): 78-80.

[2] Bai Dong, Peng Juan, Yan Han et al. HPLC-UV-ELSD combined detection of tauroursodeoxycholic acid in Xiongxiang Capsules. Liaoning Journal of Traditional Chinese Medicine. 2010; 37(6): 1108-1109.

[3] Zhao Yong, Zan Lixia, Sun Wenji. Determination of tauroursodeoxycholic acid and taurochenodeoxycholic acid in bear bile powder by HPLC-ELSD method. Journal of Pharmaceutical Analysis. 2006; 26(1): 127-129.

[4] Ba Guoji, Pan Feng, Jiang Wanlang. HPLC method to determine the content of tauroursodeoxycholic acid in Biyuan spray. Journal of Pharmaceutical Analysis. 2003; 23(2): 114-115. 